\title{
Restricted robust uniform matroid maximization under interval uncertainty
}

\author{
H. Yaman • O. E. Karaşan • M. Ç. Pınar
}

Received: 16 June 2004 / Accepted: 26 April 2006 /

Published online: 3 June 2006

(C) Springer-Verlag 2006

\begin{abstract}
For the problem of selecting $p$ items with interval objective function coefficients so as to maximize total profit, we introduce the $r$-restricted robust deviation criterion and seek solutions that minimize the $r$-restricted robust deviation. This new criterion increases the modeling power of the robust deviation (minmax regret) criterion by reducing the level of conservatism of the robust solution. It is shown that $r$-restricted robust deviation solutions can be computed efficiently. Results of experiments and comparisons with absolute robustness, robust deviation and restricted absolute robustness criteria are reported.
\end{abstract}

Keywords Uniform matroid · Robust optimization · Interval data

Mathematics Subject Classification (2000) 90C10 • 90C27 · 90C47

\section{Introduction}

The purpose of this paper is to introduce a new measure of robustness called the $r$-restricted robust deviation, and investigate its applicability within the context of a well-known problem from combinatorial optimization,namely the problem

This work is supported by a grant from Turkish Academy of Science(TUBA).

H. Yaman · O. E. Karaşan · M. Ç. Pınar $(\varangle)$

Department of Industrial Engineering,

Bilkent University, Ankara, Turkey

e-mail: mustafap@bilkent.edu.tr

H. Yaman

e-mail: hyaman@bilkent.edu.tr

O. E. Karaşan

e-mail: karasan@bilkent.edu.tr 
of selecting $p$ items out of $n$ so as to maximize total profit. It is assumed that the profit coefficients in the objective function are uncertain and can assume any value within a finite interval. This type of problems has been introduced and investigated in a series of papers and a monograph by Kouvelis and $\mathrm{Yu}$ (and coauthors); see [9]. A sample of subsequent contributions include [2,7,10,12,14]. The unifying theme is the treatment of well-known combinatorial optimization problems with imprecise data. It is usually assumed that the data behave according to some scenarios, or that the data elements can assume any value in some interval. As new concepts of optimality were needed for such situations, the contributors proposed to seek a solution that minimizes (resp. maximizes) some measure of worst performance, i.e., a solution that makes the maximum (resp. the minimum) of a performance measure minimum (resp. maximum). This paradigm gave rise to the concepts of absolute robustness (also known as minmax criterion), and robust deviation (or minmax regret criterion). There are different definitions of robust optimization problems in the literature [3-6,8] although usually these approaches also boil down to a minmax or maxmin optimization context. The approach by Ben-Tal and Nemirovski $[3,4]$ extensively studied convex optimization problems under ellipsoidal data uncertainty although it is not immediately applicable to discrete optimization. Another model by Bertsimas and Sim $[5,6]$ adopts the interval model of uncertainty and proposes a restricted version of absolute robustness criterion although this connection is overlooked by the authors. They limit the conservatism of the robust solution by arguing that it is quite unlikely that all data elements assume their worst possible values simultaneously whereas both the absolute robustness and robust deviation criteria seek solutions for such a contingency.

In the scenario model of uncertainty, both with the absolute robustness criterion and robust deviation criterion, even the simplest combinatorial optimization problems become intractable. Under the interval model of uncertainty, a positive result about tractability was obtained by Averbakh [2], and improved by Conde [7], which constitute the starting point of the present paper. Inspired by the work of Bertsimas and Sim, we develop a restricted version of the robust deviation criterion using the problem of Averbakh and Conde, namely, the problem of selecting $p$ elements out of $n$ elements so as to maximize total profit. This problem is also known as the problem of maximization over a uniform matroid and is solvable by a simple procedure in $O(n)$ time (see [7]) if data are known with certainty. Under the interval model of uncertainty of the objective function coefficients, and using the robust deviation criterion, Averbakh gave a polynomial time algorithm, which was improved recently by Conde. In the present paper, we derive the $r$-restricted robust deviation version of the problem in the aim of limiting conservatism, and show that it is polynomially solvable.

The rest of the paper is organized as follows. In Sect. 2 we give a background on robustness criteria for the maximization problem over uniform matroid. In Sect. 3 we formulate the $r$-restricted robust problem, and establish its polynomial solvability. Section 4 is devoted to numerical results. 


\section{Background}

Let a discrete ground set $N$ of $n$ items be given. Denote by $F$ the set of feasible solutions. Consider problem $P$ defined as $\max _{x \in F} \sum_{i \in N} c_{i} x_{i}$. It is assumed that the objective function coefficient of $i \in N$ denoted by $c_{i}$ is not known with certainty, but it is known to take a value in the interval $\left[l_{i}, u_{i}\right]$. For $i \in N$, we define $w_{i}=u_{i}-l_{i}$ and assume that $w_{i} \geq 0$. Let $S$ denote the set of scenarios, i.e, the Cartesian product of all intervals. For $s \in S, c(s)$ denotes the vector of objective function coefficients in scenario $s$. Following Kouvelis and Yu [9], we have the following definitions.

Definition 1 The worst performance for $x \in F$ is $a_{x}=\min _{s \in S} c(s)^{\mathrm{T}} x$. A solution $x^{*}$ is called an absolute robust solution if $x^{*} \in \operatorname{argmax}_{x \in F} a_{x}$. The problem of finding an absolute robust solution is called the absolute robust problem (AR). The robust deviation for $x \in F$ is $d_{x}=\max _{s \in S}\left(\max _{y \in F} c(s)^{\mathrm{T}} y-c(s)^{\mathrm{T}} x\right)$. A solution $x^{*}$ is called a robust deviation solution if $x^{*} \in \operatorname{argmin}_{x \in F} d_{x}$. The problem of finding a robust deviation solution is called the robust deviation problem (RD).

Problem AR can be solved easily by solving problem $P$ for the scenario $s$ such that $c(s)_{i}=l_{i}$ for all $i \in N$. Problem RD has received more attention. It has indeed led to interesting problems from the modeling and computational point of view (see e.g. $[1,2,7,11,12,14,16])$. Both robustness concepts are based on a worst case analysis. They assume that the worst scenario is likely to happen. However, in most practical situations, the probability that all parameters simultaneously take their worst possible values may be very small. One may be interested in solutions that are robust when at most a fixed number of parameters take their worst possible values. Now, following Bertsimas and Sim $[5,6]$ we give our definition of restricted robust problems. For $1 \leq r \leq n$, define $S(r)=\left\{s \in S: c(s)_{i}<u_{i}\right.$ for at most $r$ items $\}$.

Definition 2 The r-restricted worst performance is $a_{x}^{r}=\min _{s \in S(r)} c(s)^{\mathrm{T}} x$ for $x \in F$. A solution $x^{*}$ is called an r-restricted absolute robust solution if $x^{*} \in$ $\operatorname{argmax}_{x \in F} a_{x}^{r}$. The problem of finding an r-restricted absolute robust solution is called the r-restricted absolute robust problem ( $r$-RAR).

In cases where the feasible set $F$ of the generic problem $P$ is described by affine inequalities or is a discrete set, Bertsimas and Sim $[5,6]$ show that whenever $P$ is polynomially solvable, so is $r$-RAR.

Now, we can define the problem of interest for the present paper.

Definition 3 For $x \in F, d_{x}^{r}=\max _{s \in S(r)}\left(\max _{y \in F} c(s)^{\mathrm{T}} y-c(s)^{\mathrm{T}} x\right)$ is the r-restricted robust deviation. A solution $x^{*}$ is called an $r$-restricted robust deviation solution if $x^{*} \in \operatorname{argmin}_{x \in F} d_{x}^{r}$. The problem of finding an r-restricted robust deviation solution is called the r-restricted robust deviation problem ( $r$-RRD).

It is easy to see the following relationship between these problems:

Proposition 1 For $x \in F, a_{x}^{n}=a_{x}$ and $d_{x}^{n}=d_{x}$. 
This proposition has the following consequences: AR is a special case of $r$-RAR. Once we know that $r$-RAR is polynomially solvable, AR is also polynomially solvable. Similarly, RD is a special case of $r$-RRD. Hence, if we know that RD is NP-hard, we can immediately conclude that $r$-RRD is NP-hard. Unfortunately, the robust deviation counterpart of even some easy problems are known to be NP-hard. Examples are shortest path problem (see [16]) and minimum spanning tree problem (see [1]). So the $r$-RRD counterparts of these problems are also NP-hard.

Averbakh [2] proved that RD in the setting of maximization over a uniform matroid is polynomially solvable. One of the main questions in this paper is whether $r$-RRD in the same setting is also polynomially solvable. Before answering this question, we derive the formulations of RD and $r$-RAR for maximization over a uniform matroid. Let $p \leq n$ and $F=\left\{x \in\{0,1\}^{n}: \sum_{i \in N} x_{i}=p\right\}$. The deterministic problem is defined as $\max _{x \in F} \sum_{i \in N} c_{i} x_{i}$.

Problem AR can be solved by taking $c_{i}=l_{i}$ for all $i \in N$ in the above formulation. To be able to formulate $\mathrm{RD}$, we need the following result which was shown in [2,7]: $d_{x}=\max _{y \in F}\left(\sum_{i \in N} u_{i}-w_{i} x_{i}\right) y_{i}-\sum_{i \in N} l_{i} x_{i}$. As $\operatorname{conv}(F)=\{y \in$ $\left.\mathbb{R}_{+}^{n}: \sum_{i \in N} y_{i}=p, y_{i} \leq 1 \quad \forall i \in N\right\}$, by the Strong Duality Theorem (SDT) of Linear Programming (LP), we have $d_{x}=\min _{(\lambda, \gamma) \in \Lambda}\left(p \lambda+\sum_{i \in N} \gamma_{i}\right)-\sum_{i \in N} l_{i} x_{i}$ where $\Lambda=\left\{(\lambda, \gamma) \in \mathbb{R}^{n+1}: \lambda+\gamma_{i} \geq u_{i}-w_{i} x_{i}\right.$ and $\left.\gamma_{i} \geq 0 \quad \forall i \in N\right\}$. Therefore $\mathrm{RD}$ is formulated as:

$$
\begin{array}{lll}
\text { (RD) } \min & p \lambda+\sum_{i \in N} \gamma_{i}-\sum_{i \in N} l_{i} x_{i} & \\
\text { s.t. } & x \in F & \\
& \lambda+\gamma_{i} \geq u_{i}-w_{i} x_{i}, & \forall i \in N \\
& \gamma_{i} \geq 0, & \forall i \in N .
\end{array}
$$

The above problem was shown to be polynomially solvable in [2,7].

Let $1 \leq r \leq n$ and $Z(r)=\left\{z \in\{0,1\}^{n}: \sum_{i \in N} z_{i} \leq r\right\}$. For $x \in F$, the $r$-restricted worst performance can be computed as $a_{x}^{r}=\sum_{i \in N} u_{i} x_{i}-\max _{z \in Z(r)}$ $\sum_{i \in N} w_{i} z_{i} x_{i}$. As $\operatorname{conv}\left(Z_{r}\right)=\left\{z \in \mathbb{R}_{+}^{n}: \sum_{i \in N} z_{i} \leq r, z_{i} \leq 1 \quad \forall i \in N\right\}$, by the SDT of LP, we have $a_{x}^{r}=\sum_{i \in N} u_{i} x_{i}-\min _{(\mu, \gamma) \in \Gamma} \mu r+\sum_{i \in N} \gamma_{i}$ where $\Gamma=\{(\mu, \gamma) \in$ $\mathbb{R}_{+}^{n+1}: \mu+\gamma_{i} \geq w_{i} x_{i}$. Hence $r$-RAR is formulated as:

$$
\begin{array}{cll}
(r \text {-RAR }) \max & \sum_{i \in N} u_{i} x_{i}-\mu r-\sum_{i \in N} \gamma_{i} \\
\text { s.t. } \quad & x \in F \\
& \mu+\gamma_{i} \geq w_{i} x_{i}, \quad \forall i \in N \\
& \mu \geq 0, \quad \forall i \in N .
\end{array}
$$

This problem is also polynomially solvable [5].

To end this section, we relate the four robust problems for maximization over a uniform matroid with a result stronger than Proposition 1. 
Proposition 2 For $x \in F, a_{x}^{r}=a_{x}^{n}=a_{x}$ for all $r \geq p$ and $d_{x}^{r}=d_{x}^{n}=d_{x}$ for all $r \geq \min \{p, n-p\}$.

Proof As $S(1) \subseteq S(2) \subseteq \cdots \subseteq S(n)=S$, for $x \in F$, we have $a_{x}^{1} \geq a_{x}^{2} \geq \cdots \geq$ $a_{x}^{n}=a_{x}$ and $d_{x}^{1} \leq d_{x}^{2} \leq \cdots \leq d_{x}^{n}=d_{x}$. For $r>p$, let $a_{x}^{r}=\min _{s \in S(r)} c(s)^{\mathrm{T}} x=$ $c\left(s^{*}\right)^{\mathrm{T}} x$. Construct scenario $s^{\prime}$ as follows: for $i \in N$, if $c\left(s^{*}\right)_{i}<u_{i}$ and $x_{i}=0$, then $c\left(s^{\prime}\right)_{i}=u_{i}$ and $c\left(s^{\prime}\right)_{i}=c\left(s^{*}\right)_{i}$ otherwise. Then $s^{\prime} \in S(p)$ and $c\left(s^{*}\right)^{\mathrm{T}} x=c\left(s^{\prime}\right)^{\mathrm{T}} x$. As $a_{x}^{p}=\min _{s \in S(p)} c(s)^{\mathrm{T}} x \leq c\left(s^{\prime}\right)^{\mathrm{T}} x$, we have $a_{x}^{p} \leq a_{x}^{r}$. We also know that $a_{x}^{p} \geq a_{x}^{r}$ since $r>p$. So $a_{x}^{p}=a_{x}^{r}$ for all $r>p$. Let $\bar{p}=\min \{p, n-p\}$. For $r>\bar{p}$, let $d_{x}^{r}=\max _{s \in S(r)}\left(\max _{y \in F} c(s)^{\mathrm{T}} y-c(s)^{\mathrm{T}} x\right)=c\left(s^{*}\right)^{\mathrm{T}} y^{*}-c\left(s^{*}\right)^{\mathrm{T}} x$. Construct scenario $s^{\prime}$ as follows: for $i \in N$, if $c\left(s^{*}\right)_{i}<u_{i}$ and $x_{i}=0$ or $y_{i}=1$, then $c\left(s^{\prime}\right)_{i}=u_{i}$ and $c\left(s^{\prime}\right)_{i}=c\left(s^{*}\right)_{i}$ otherwise. Then $s^{\prime} \in S(\bar{p})$ and $c\left(s^{*}\right)^{\mathrm{T}} y^{*}-c\left(s^{*}\right)^{\mathrm{T}} x \leq$ $c\left(s^{\prime}\right)^{\mathrm{T}} y^{*}-c\left(s^{\prime}\right)^{\mathrm{T}} x$. So $d_{x}^{r} \leq c\left(s^{\prime}\right)^{\mathrm{T}} y^{*}-c\left(s^{\prime}\right)^{\mathrm{T}} x$. As $d_{x}^{\bar{p}} \geq c\left(s^{\prime}\right)^{\mathrm{T}} y^{*}-c\left(s^{\prime}\right)^{\mathrm{T}} x$, we have $d_{x}^{r} \leq d_{x}^{\bar{p}}$. Together with $d_{x}^{r} \geq d_{x}^{\bar{p}}$, this implies that $d_{x}^{r}=d_{x}^{\bar{p}}$ for $r>\bar{p}$.

\section{Structural results, formulation and solvability status}

Now we present a MIP formulation of the $r$-restricted robust problem.

Proposition 3 For $x \in F$,

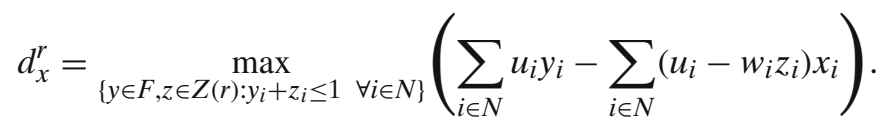

Proof Let $s^{*}$ and $y^{*}$ be such that $d_{x}^{r}=c\left(s^{*}\right)^{\mathrm{T}} y^{*}-c\left(s^{*}\right)^{\mathrm{T}} x$ and define $z^{*}$ and $v^{*}$ as follows: for $i \in N$, if $c\left(s^{*}\right)_{i}<u_{i}$ then $z_{i}^{*}=1$ and $v_{i}^{*}=u_{i}-c\left(s^{*}\right)_{i}$ and if $c\left(s^{*}\right)_{i}=u_{i}$ then $z_{i}^{*}=0$ and $v_{i}^{*}=0$. Consider the scenario $s^{\prime}$ such that $c\left(s^{\prime}\right)_{i}=l_{i}$ if $z_{i}^{*}=1$ and $y_{i}^{*}=0$ and $c\left(s^{\prime}\right)_{i}=u_{i}$ otherwise. For a $0-1$ vector $x$, define its support $I(x)=\left\{i \in N: x_{i}=1\right\}$. Then

$$
\begin{aligned}
c\left(s^{*}\right)^{\mathrm{T}} y^{*}-c\left(s^{*}\right)^{\mathrm{T}} x & =\sum_{i \in I\left(y^{*}\right) \backslash I(x)} c\left(s^{*}\right)_{i}-\sum_{i \in I(x) \backslash I\left(y^{*}\right)} c\left(s^{*}\right)_{i} \\
& =\sum_{i \in I\left(y^{*}\right) \backslash I(x)}\left(u_{i}-v_{i}^{*} z_{i}^{*}\right)-\sum_{i \in I(x) \backslash I\left(y^{*}\right)}\left(u_{i}-v_{i}^{*} z_{i}^{*}\right) \\
& \leq \sum_{i \in I\left(y^{*}\right) \backslash I(x)} u_{i}-\sum_{i \in I(x) \backslash I\left(y^{*}\right)}\left(u_{i}-w_{i} z_{i}^{*}\right) \\
& =c\left(s^{\prime}\right)^{\mathrm{T}} y^{*}-c\left(s^{\prime}\right)^{\mathrm{T}} x \\
& \leq \max _{y \in F} c\left(s^{\prime}\right)^{\mathrm{T}} y-c\left(s^{\prime}\right)^{\mathrm{T}} x \\
& \leq \max _{s \in S(r)}\left(\max _{y \in F} c(s)^{\mathrm{T}} y-c(s)^{\mathrm{T}} x\right) .
\end{aligned}
$$

As $d_{x}^{r}=\max _{s \in S(r)}\left(\max _{y \in F} c(s)^{\mathrm{T}} y-c(s)^{\mathrm{T}} x\right)$, all above inequalities are satisfied at equality. Hence, there exists an optimal solution where $y_{i}+z_{i} \leq 1$ for all $i \in N$ 
and the objective function coefficient of item $i$ is at its lower bound $l_{i}$ whenever $z_{i}=1$.

Therefore, for a given $x \in F$, its $r$-robust deviation can be computed as $d_{x}^{r}=f_{x}^{r}-\sum_{i \in N} u_{i} x_{i}$ where

$$
\begin{aligned}
f_{x}^{r}=\max & \sum_{i \in N}\left(u_{i} y_{i}+w_{i} x_{i} z_{i}\right) \\
\text { s.t. } & \sum_{i \in N} y_{i}=p \\
& \sum_{i \in N} z_{i} \leq r \\
& y_{i}+z_{i} \leq 1, \quad \forall i \in N \\
& y_{i}, z_{i} \in\{0,1\}, \quad \forall i \in N .
\end{aligned}
$$

Theorem 1 Problem r-RRD can be formulated as follows:

$$
\begin{aligned}
& (r-\mathrm{RRD}) \min p \lambda+r \mu+\sum_{i \in N} \gamma_{i}-\sum_{i \in N} u_{i} x_{i} \\
& \text { s.t. } x \in F \\
& \lambda+\gamma_{i} \geq u_{i}, \quad \forall i \in N \\
& \mu+\gamma_{i} \geq w_{i} x_{i}, \quad \forall i \in N \\
& \mu \geq 0 \\
& \gamma_{i} \geq 0, \quad \forall i \in N \text {. }
\end{aligned}
$$

Proof Let $F_{x}^{r}=\left\{(y, z) \in \mathbb{R}_{+}^{2 n}:(1)-(4)\right\}$. We first show that $\operatorname{conv}\left(F_{x}^{r}\right)=\{(y, z) \in$ $\left.\mathbb{R}_{+}^{2 n}:(1)-(3)\right\}$. Let $H$ be the matrix of left hand side coefficients of constraints $\sum_{i \in N} y_{i} \leq p,-\sum_{i \in N} y_{i} \leq-p$, (2) and (3). Matrix $H$ is totally unimodular (TU) if each collection of columns of $H$ can be split into two so that the sum of the columns in one minus the sum of the columns in the other is a vector with entries in $\{0,1,-1\}$ (see Schrijver [13], Theorem 19.3). Let $H_{1}=\left\{h_{1}^{1}, h_{2}^{1}, \ldots, h_{n}^{1}\right\}$ be the set of first $n$ columns of $H$ and let $H_{2}=\left\{h_{1}^{2}, h_{2}^{2}, \ldots, h_{n}^{2}\right\}$ be the set of last $n$ columns of $H$. Given a set $C$ (we can consider sets instead of collections, since a repeated column is put in two different parts) of columns of $H$, we can partition $C$ into two parts $C_{1}$ and $C_{2}$ such that the difference of the sum of the columns in $C_{1}$ and the sum of the columns in $C_{2}$ has components $0,+1$ and -1 as follows. Without loss of generality, suppose that $\{1,2, \ldots, k\}$ is the set of indices $i$ such that $h_{i}^{1} \in C$ and $h_{i}^{2} \in C,\{k+1, k+2, \ldots, l\}$ is the set of indices $i$ such that $h_{i}^{1} \in C$ and $h_{i}^{2} \notin C$ and $\{l+1, l+2, \ldots, m\}$ is the set of indices $i$ such that $h_{i}^{1} \notin C$ and $h_{i}^{2} \in C$. For $j=1,2, \ldots, k$, if $j$ is odd, put $h_{j}^{1}$ to $C_{1}$ and $h_{j}^{2}$ to $C_{2}$ and if $j$ is even, put $h_{j}^{1}$ to $C_{2}$ and $h_{j}^{2}$ to $C_{1}$. For $j=1,2, \ldots, l-k$, if $j$ is odd, put $h_{j+k}^{1}$ to $C_{2}$ and if $j$ is even, put $h_{j+k}^{1}$ to $C_{1}$. For $j=1,2, \ldots, m-l$, if $j$ is odd, put $h_{j+l}^{2}$ to $C_{1}$ and if $j$ is even, put $h_{j+l}^{2}$ to $C_{2}$. 
As $H$ is TU and the right hand side vector is integral, $\left\{(y, z) \in \mathbb{R}_{+}^{2 n}:(1)-(3)\right\}$ is an integral polytope (see Schrijver [13], Corollary 19.2a). As a consequence of this observation, for a given $x$, the $r$-restricted robust deviation can be computed by solving an LP. Associate dual variables $\lambda$ to constraint (1), $\mu$ to (2) and $\gamma_{i}$ to (3) for all $i \in N$. Then SDT of LP implies that $f_{x}^{r}=\min p \lambda+r \mu+\sum_{i \in N} \gamma_{i}$ under constraints (6)-(9).

It is easy to verify that when $r=p$ the formulation of Theorem 1 is transformed to RD of [7].

Now, we show that there exists an optimal solution to $r$-RRD with a reduced search space for $\lambda$ and $\mu$. Let $L=\left\{l_{1}, l_{2}, \ldots, l_{n}\right\}, U=\left\{u_{1}, u_{2}, \ldots, u_{n}\right\}$ and $W=\left\{w_{1}, w_{2}, \ldots, w_{n}\right\}$.

Theorem 2 There exists $\left(\mathbf{x}^{*}, \lambda^{*}, \mu^{*}, \boldsymbol{\gamma}^{*}\right)$ optimal for $r$-RRD such that the following statements are true:

a. Either $\lambda^{*}-\mu^{*} \in L$ or $\mu^{*} \in W$ and $\lambda^{*} \in U$.

b. If $\mu^{*}>0$ then either $\lambda^{*} \in U$ or $\mu^{*} \in W$.

Proof Let $\left(\mathbf{x}^{*}, \lambda^{*}, \mu^{*}, \gamma^{*}\right)$ be an extreme point optimal solution. Without loss of generality, assume $x_{i}^{*}=1$ for $i \in\{1, \ldots, p\}$ and $x_{i}^{*}=0$ otherwise. Then, $\gamma_{i}^{*}=\max \left\{w_{i}-\mu^{*}, u_{i}-\lambda^{*}, 0\right\}$ for $i \in\{1, \ldots, p\}$, and $\gamma_{i}^{*}=\max \left\{u_{i}-\lambda^{*}, 0\right\}$ for $i \in$ $\{p+1, \ldots, n\}$. Let $A=\left\{i \in\{1, \ldots, p\}: w_{i}-\mu^{*}>u_{i}-\lambda^{*}\right\}$. We subdivide $A$ into $A_{1}, A_{2}$ and $A_{3}$ such that $A_{1}=\left\{i \in A: w_{i}-\mu^{*}>0\right\}, A_{2}=\left\{i \in A: w_{i}-\mu^{*}=0\right\}$ and $A_{3}=\left\{i \in A: w_{i}-\mu^{*}<0\right\}$. Let $B=\left\{i \in\{1, \ldots, p\}: w_{i}-\mu^{*}=u_{i}-\lambda^{*}\right\}$ subdivided into $B_{1}=\left\{i \in B: w_{i}-\mu^{*}=u_{i}-\lambda^{*}>0\right\}, B_{2}=\{i \in B$ : $\left.w_{i}-\mu^{*}=u_{i}-\lambda^{*}=0\right\}$, and $B_{3}=\left\{i \in B: w_{i}-\mu^{*}=u_{i}-\lambda^{*}<0\right\}$. We also have $C=\left\{i \in\{1, \ldots, p\}: w_{i}-\mu^{*}<u_{i}-\lambda^{*}\right\}$ partitioned into $C_{1}=\left\{i \in C: u_{i}-\lambda^{*}>0\right\}$, $C_{2}=\left\{i \in C: u_{i}-\lambda^{*}=0\right\}$, and $C_{3}=\left\{i \in C: u_{i}-\lambda^{*}<0\right\}$. Similarly, let $D=\left\{i \in\{p+1, \ldots, n\}: u_{i}-\lambda^{*}>0\right\}, E=\left\{i \in\{p+1, \ldots, n\}: u_{i}-\lambda^{*}=0\right\}$ and $F=\left\{i \in\{p+1, \ldots, n\}: u_{i}-\lambda^{*}<0\right\}$.

First for the proof of part a, assume neither $\lambda^{*}-\mu^{*} \in L$ nor $\mu^{*} \in W$. This implies $A_{2}=B=\emptyset$. Now, we have $\gamma_{i}^{*}=w_{i}-\mu^{*}$ for $i \in A_{1}, \gamma_{i}^{*}=0$ for $i \in A_{3}, \gamma_{i}^{*}=u_{i}-\lambda^{*}$ for $i \in C_{1}, \gamma_{i}^{*}=u_{i}-\lambda^{*}=0$ for $i \in C_{2}, \gamma_{i}^{*}=0$ for $i \in C_{3}, \gamma_{i}^{*}=u_{i}-\lambda^{*}$ for $i \in D, \gamma_{i}^{*}=u_{i}-\lambda^{*}=0$ for $i \in E$ and finally $\gamma_{i}^{*}=0$ for $i \in F$. Let $\left(\mathbf{x}^{*}, \lambda^{*}, \mu^{*}, \gamma_{A_{1}}^{*}, \gamma_{A_{3}}^{*}, \gamma_{C_{1}}^{*}, \gamma_{C_{2}}^{*}, \gamma_{C_{3}}^{*}, \gamma_{D}^{*}, \gamma_{E}^{*}, \gamma_{F}^{*}\right)$ be the vectorial description of the current solution. For a given set $\mathcal{S}$ and some positive $\epsilon$, let $\epsilon_{\mathcal{S}}$ be a vector of dimension $|\mathcal{S}|$ with all entries equal to $\epsilon$. Then, both $\left(\mathbf{x}^{*}, \lambda^{*}, \mu^{*}+\epsilon, \gamma_{A_{1}}^{*}-\epsilon_{A_{1}}, \gamma_{A_{3}}^{*}, \gamma_{C_{1}}^{*}, \gamma_{C_{2}}^{*}, \gamma_{C_{3}}^{*}, \gamma_{D}^{*}, \gamma_{E}^{*}, \gamma_{F}^{*}\right)$ and $\left(\mathbf{x}^{*}, \lambda^{*}, \mu^{*}-\right.$ $\left.\epsilon, \gamma_{A_{1}}^{*}+\epsilon_{A_{1}}, \gamma_{A_{3}}^{*}, \gamma_{C_{1}}^{*}, \gamma_{C_{2}}^{*}, \gamma_{C_{3}}^{*}, \gamma_{D}^{*}, \gamma_{E}^{*}, \gamma_{F}^{*}\right)$ are feasible solutions of $r$-RRD for small enough $\epsilon$ which contradicts the extremality of the starting optimal solution. Now, assume neither $\lambda^{*}-\mu^{*} \in L$ nor $\lambda^{*} \in U$, i.e., $B=C_{2}=E=\emptyset$. As above, both $\left(\mathbf{x}^{*}, \lambda^{*}+\epsilon, \mu^{*}, \gamma_{A_{1}}^{*}, \gamma_{A_{2}}^{*}, \gamma_{A_{3}}^{*}, \gamma_{C_{1}}^{*}-\epsilon_{C_{1}}, \gamma_{C_{3}}^{*}, \gamma_{D}^{*}-\epsilon_{D}, \gamma_{F}^{*}\right)$ and $\left(\mathbf{x}^{*}, \lambda^{*}-\epsilon, \mu^{*}, \gamma_{A_{1}}^{*}, \gamma_{A_{2}}^{*}, \gamma_{A_{3}}^{*}, \gamma_{C_{1}}^{*}+\epsilon_{C_{1}}, \gamma_{C_{3}}^{*}, \gamma_{D}^{*}+\epsilon_{D}, \gamma_{F}^{*}\right)$ are again feasible solutions to $r$-RRD with small enough $\epsilon$. For part $b$, assume that $\mu^{*}>0$ but neither $\lambda^{*} \in U$ nor $\mu^{*} \in W$, i.e., $A_{2}=B_{2}=C_{2}=E=\emptyset$. Now, both $\left(\mathbf{x}^{*}, \lambda^{*}+\epsilon, \mu^{*}+\epsilon, \gamma_{A_{1}}^{*}-\epsilon_{A_{1}}, \gamma_{A_{3}}^{*}, \gamma_{B_{1}}^{*}-\epsilon_{B_{1}}, \gamma_{B_{3}}^{*}, \gamma_{C_{1}}^{*}-\epsilon_{C_{1}}, \gamma_{C_{3}}^{*}, \gamma_{D}^{*}-\epsilon_{D}, \gamma_{F}^{*}\right)$ 
and $\left(\mathbf{x}^{*}, \lambda^{*}-\epsilon, \mu^{*}-\epsilon, \gamma_{A_{1}}^{*}+\epsilon_{A_{1}}, \gamma_{A_{3}}^{*}, \gamma_{B_{1}}^{*}+\epsilon_{B_{1}}, \gamma_{B_{3}}^{*}, \gamma_{C_{1}}^{*}+\epsilon_{C_{1}}, \gamma_{C_{3}}^{*}, \gamma_{D}^{*}+\epsilon_{D}, \gamma_{F}^{*}\right)$ are feasible.

The solution of $r$-RRD for fixed $\lambda$ and $\mu$ boils down to minimization of a piecewise linear function as problem (3) of [7], the evaluation of which can be accomplished in $O(n)$ time. More precisely for fixed $\lambda$ and $\mu$, we solve the problem of minimizing $f(\lambda, \mu)=p \lambda+r \mu+\sum_{i \in N} \max \left\{u_{i}-\lambda, w_{i} x_{i}-\mu, 0\right\}-$ $\sum_{i \in N} u_{i} x_{i}$ under the restriction $x \in F$. Therefore, we transformed $r$-RRD into $\min _{\lambda, \mu} f(\lambda, \mu)$. We solve the latter problem by testing the critical values of $\lambda$ and $\mu$ following Theorem 2. If $\lambda \in U$ and $\mu \in W$, then we need to test $n^{2}$ values. Otherwise, $\lambda-\mu \in L$. If $\mu=0$, then $\lambda \in L$ and so can take $n$ different values. Finally, if $\mu>0$, then either $\lambda \in U$ or $\mu \in W$, resulting in additional $2 n^{2}$ values to test. We simply pick the $\lambda$ and $\mu$ values yielding the smallest objective function value. Therefore, we have

Theorem 3 Problem $r$-RRD can be solved in $O\left(n^{3}\right)$ time.

\section{Experiments}

In this section we summarize experimental evidence to the effectiveness of the $r$-restricted robust deviation criterion. The detailed results can be found in the longer version [15]. By an uncertain problem we mean that we fix $n$ and $p$ and generate for each objective function coefficient a random interval, i.e., $l_{i}$ and $u_{i}$ values for each $i$. In all experiments we generate an uncertain problem with $n=500$ randomly where we take the $l_{i}$ values uniformly distributed in the interval $(-10,000 ; 10,000), w_{i}$ values uniformly distributed in the interval $(0 ; 2,000)$ and we obtain $u_{i}$ values by simply adding $w_{i}$ to $l_{i}$ for each $i$. An instance of an uncertain problem corresponds to a random scenario of objective function coefficients within the prespecified interval.

Our first experiment compares the relative performances of the four robustness measures of this paper. We summarize this experiment in Fig. 1 and Table 1. For Fig. 1, we first generate an uncertain problem with $p=250$. Then we randomly generate what we call "extreme" problem instances in order to be able to observe a distinct behavior of the $r$-robust and the robust deviation solutions, which is usually impossible to obtain without such extreme instances. For a fixed $r$, we take objective function coefficients at their lower bounds with probability equal to $\frac{r}{n}$ and at their upper bounds with probability equal to $1-\frac{r}{n}$. We repeat this 50 times, thus obtaining 50 problem instances for fixed $p$ and $r$. Then we compute the $r$-restricted robust solution and the robust deviation solution. We calculate the deviations of these solutions from the optimal values of each of the 50 instances. We take the average of these 50 observations for both the $r$-robust solution and the robust deviation solution, respectively. Repeating this for values of $r$ ranging from 1 to $p$, we obtain an entire plot. We observe that for small values of $r$, the $r$-robust solution is somewhat more robust in such extreme scenarios compared to the robust deviation solution whereas after a certain $r$ value the two solutions behave identically. 


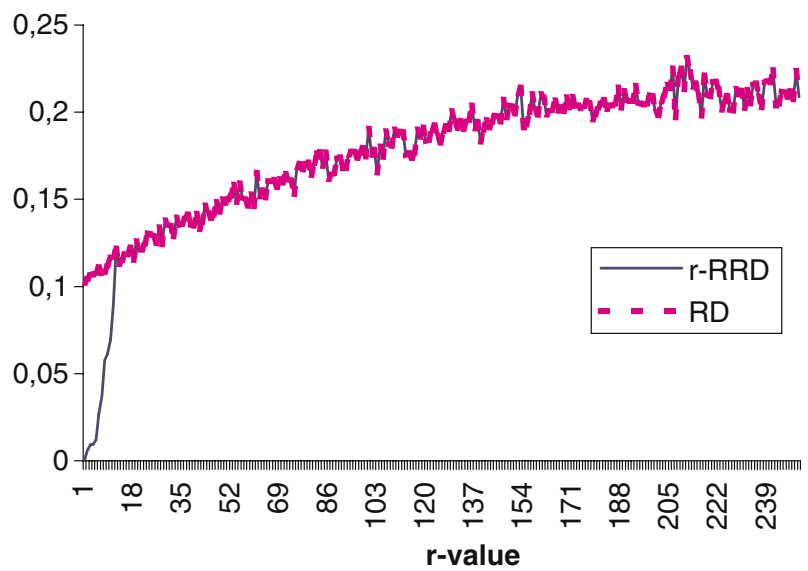

Fig. 1 Average percentage deviations of robust deviation and $r$-robust solutions from the optimal value for extreme problem instances: $p=250$

Table $1 \ell_{1}$ and $\ell_{\infty}$ norms of error vectors for three different robustness criteria averaged over 50 uncertain problems tested against 500 normally distributed randomly generated instances

\begin{tabular}{|c|c|c|c|c|c|c|}
\hline \multirow[t]{2}{*}{$p, r$} & \multicolumn{3}{|l|}{$\ell_{1}$ norm } & \multicolumn{3}{|c|}{$\ell_{\infty}$ norm } \\
\hline & $r$-RRD & $r$-RAR & $\mathrm{AR}$ & $r$-RRD & $r$-RAR & AR \\
\hline 50,10 & 1.205 & 2.053 & 2.242 & 0.007 & 0.010 & 0.010 \\
\hline 50,25 & 1.173 & 1.474 & 2.242 & 0.007 & 0.008 & 0.010 \\
\hline 50,40 & 1.173 & 1.897 & 2.242 & 0.007 & 0.009 & 0.010 \\
\hline 100,20 & 0.596 & 1.091 & 1.150 & 0.004 & 0.005 & 0.005 \\
\hline 100,50 & 0.596 & 0.725 & 1.150 & 0.004 & 0.004 & 0.005 \\
\hline 100,80 & 0.596 & 0.936 & 1.150 & 0.004 & 0.005 & 0.005 \\
\hline 200,40 & 0.396 & 0.663 & 0.757 & 0.003 & 0.003 & 0.004 \\
\hline 200,100 & 0.396 & 0.491 & 0.757 & 0.003 & 0.003 & 0.004 \\
\hline 200,160 & 0.396 & 0.625 & 0.757 & 0.003 & 0.003 & 0.004 \\
\hline 250,50 & 0.421 & 0.695 & 0.855 & 0.003 & 0.003 & 0.004 \\
\hline 250,125 & 0.421 & 0.514 & 0.855 & 0.003 & 0.003 & 0.004 \\
\hline 250,200 & 0.421 & 0.685 & 0.855 & 0.003 & 0.003 & 0.004 \\
\hline
\end{tabular}

In Table 1, we compare the performances of the $r$-robust deviation solution for a fixed value of $r$, the $r$-restricted robust absolute solution using the same value of $r$, and the absolute robust solution of Definition 1 for 50 uncertain problems. For each uncertain problem, fixing $p$ and $r$ we first compute these three solutions. Then we generate 500 random instances. We find the optimal value for each of the 500 problem instances, and compute the percentage error in objective function value corresponding to each of the three solutions with respect to the random instance's optimal value. We compute the $\ell_{1}$ and $\ell_{\infty}$ norms of this error vector of dimension 500. These results were obtained by generating the objective function coefficients according to a Normal law where the interval $\left[l_{i}, u_{i}\right]$ for coefficient $i$ corresponds to a $95 \%$-quantile. We repeat 


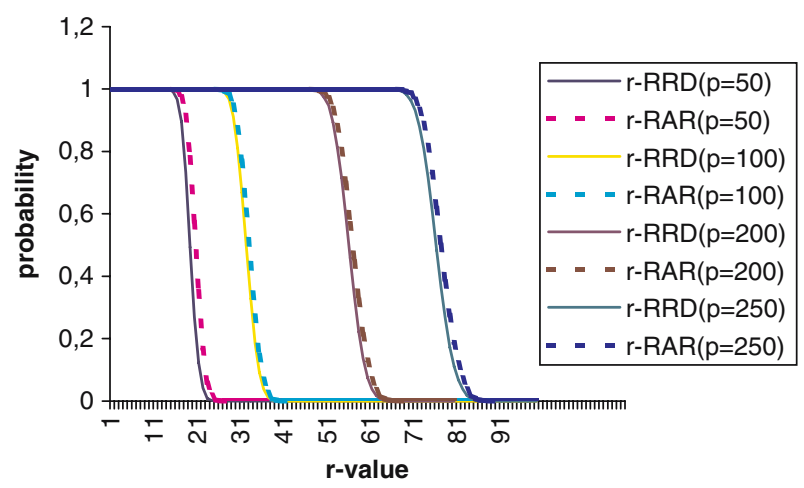

Fig. 2 Empirical probability of unsatisfactory performance using normally distributed objective function coefficients as a function of $r$

this procedure for 50 randomly generated uncertain problems. Each entry of Table 1 corresponds to the mean value of these $\ell_{1}$ and $\ell_{\infty}$ norms of percentage errors over 50 uncertain problems. The results clearly show a superiority of the $r$-restricted robust deviation solution to other measures.

The probability (under an assumed distribution of the objective function coefficients) that the robust solution fails to give a satisfactory performance is also an important determinant of robustness. The smaller this probability, the higher the protection offered by the $r$-restricted robust deviation solution. Bounds on this probability are obtained in $[5,6]$ under some general assumptions on the distribution of random parameters. An unsatisfactory performance in this context is defined as the occurrence of an objective function value at the $r$-restricted robust deviation solution smaller than the $r$-restricted worst performance (as in Definition 2) of the same solution. The reason for using Definition 2 in place of Definition 3 is that we are interested in an absolute performance in this experiment. Hence, in our second experiment we compute an empirical probability of unsatisfactory performance for the $r$-restricted robust deviation solution as a function of $r$ for a problem with fixed $n$ and $p$. For comparison, this empirical probability is also computed for the $r$-restricted absolute robust solution of Definition 2.

We randomly generate uncertain problems with $p=50,100,200$ and 250 and solve the corresponding $r$-RAR and the $r$-RRD. Then, we generate 10,000 random objective function vectors and count the occurrences of unsatisfactory trials for all robust solutions. This is repeated for increasing values of $r$ from 1 to $p$. It is certainly beneficial from a robustness point of view to choose $r$ in the range where the probability of unsatisfactory performance vanishes. The results summarized in Fig. 2 show that the critical value of $r$ (where the probability of unsatisfactory performance vanishes) for a fixed problem depends on $p$ as follows. For larger $p$, e.g., $p=200,250$, the critical value is situated at a value $\alpha p$ where $\alpha$ is slightly above $1 / 3$, e.g. 0.36 or 0.37 . This critical value becomes somewhat larger as $p$ gets smaller. The performances of the $r$-restricted absolute 
robust solution and that of the $r$-restricted robust deviation solutions are quite close, with a slight superiority of the $r$-restricted robust deviation solution.

In conclusion, the experimental results revealed that the $r$-restriction of the robust deviation solution, while less conservative, does not lead to a decrease in robustness and shows a superior behavior to previously proposed robustness concepts.

\section{References}

1. Aron, I.D., Van Hentenryck, P.: On the complexity of the robust spanning tree problem with interval data. Oper. Res. Lett. 32: 36-40 (2004)

2. Averbakh, I.: On the complexity of a class of combinatorial optimization problems with uncertainty. Math. Prog. 90: 263-272 (2001)

3. Ben-Tal, A., Nemirovski, A.: Robust convex optimization. Math. OR. 23: 769-805 (1998)

4. Ben-Tal, A., Nemirovski, A.: Lectures on modern convex optimization: analysis, algorithms and engineering applications. SIAM-MPS, Philadelphia (2000)

5. Bertsimas, D., Sim, M.: Robust discrete optimization and network flows. Math. Prog. B 98: 49-71 (2003)

6. Bertsimas, D., Sim, M.: The price of robustness. Oper. Res. 52: 35-53 (2004)

7. Conde, E.: An improved algorithm for selecting $\mathrm{p}$ items with uncertain returns according to the minmax-regret criterion. Math. Prog. 100: 345-353 (2004)

8. El-Ghaoui, L., Lebret, H.: Robust solutions to least squares problems under uncertain data matrices. SIAM J. Matrix Anal. Appl. 18: 1035-1064 (1997)

9. Kouvelis, P., Yu, G.: Robust discrete optimization and applications. Kluwer, Boston (1997)

10. Mausser, H.E., Laguna, M.: A new mixed integer formulation for the maximum regret problem. Int. Trans. Oper. Res. 5(5): 389-403 (1998)

11. Montemanni, R., Gambardella, L.M.: A branch and bound algorithm for the robust spanning tree problem with interval data. Eur. J. Oper. Res. 161: 771-729 (2005)

12. Montemanni, R., Gambardella, L.M., Donati, A.V.: A branch and bound for the robust shortest path problem with interval data. Oper. Res. Lett. 32: 225-232 (2004)

13. Schrijver, A.: Theory of linear and integer programming. Wiley, New York (1987)

14. Yaman, H., Karaşan, O.E., Pınar, M.Ç.: The robust spanning tree problem with interval data. Oper. Res. Lett. 29: 31-40 (2001)

15. Yaman, H., Karaşan, O.E., Pınar, M.Ç.: Restricted robust optimization for maximization over uniform matroid with interval data uncertainty. Technical report, Bilkent University, Ankara, Turkey, www.ie.bilkent.edu.tr/ ${ }^{\sim}$ mustafap/pubs/psecrev.pdf (2005)

16. Zielinski, P.: The computational complexity of the relative robust short path problem with interval data. Eur. J. Oper. Res. 158: 570-576 (2004) 